\section{ARF in the critically ill: CRRT associated with better long-term outcomes than IHD}

Acute renal failure (ARF) can develop in critically ill patients as a result of multiple organ failure. Currently, a considerable proportion of surviving ARF patients are dependent on dialysis at discharge, having developed irreversible renal dysfunction. It is unclear whether the treatment modality used during intensive care affects renal recovery. To address this issue, Bell et al. conducted a retrospective study of 2,202 patients with ARF who were treated with either continuous renal replacement therapy (CRRT) or intermittent hemodialysis (IHD) in 32 Swedish intensive care units. Duration of follow-up ranged from 3 months to 10 years.

In total, 1,100 patients died within 90 days of initial dialysis. No association was found between dialysis modality and 90-day mortality. Of the 90-day survivors, 944 had received CRRT and 158 had received IHD. The risk of end-stage renal disease (ESRD) was considerably higher in 90-day survivors treated with IHD than in those treated with CRRT (adjusted odds ratio $2.60,95 \% \mathrm{Cl} 1.50-4.30$ ); however, the trend towards a higher risk of ESRD with IHD decreased with increasing duration of follow-up. Among the 90-day survivors who did develop ESRD, the risk of death was markedly higher in patients treated with IHD than in those treated with CRRT (hazard ratio $2.29,95 \% \mathrm{Cl} 1.27-4.13)$. On the basis of these results, the authors conclude that the use of CRRT for ARF in critically ill patients reduces the risk of chronic renal failure.

Original article Bell M et al. (2007) Continuous renal replacement therapy is associated with less chronic renal failure than intermittent haemodialysis after acute renal failure. Intensive Care Med 33: 773-780

\section{N-terminal probrain natriuretic peptide level predictive of mortality in ESRD}

$\mathrm{N}$-terminal probrain natriuretic peptide (NTproBNP) is a well established prognostic marker in patients with heart failure. Given that cardiovascular disease is a major cause of death in people with end-stage renal disease (ESRD), it has been suggested that NT-proBNP measurement might facilitate the identification of patients in this population who are at high risk of cardiovascular events. Now, Madsen et al. have demonstrated that NT-proBNP is prognostic for mortality in maintenance hemodialysis patients.

On study entry, left ventricular hypertrophy was detected in $36.7 \%$ of the 109 study participants; $81.7 \%$ of the study population was receiving medication for hypertension. Serum levels of NT-proBNP were measured both before and after dialysis. Compared with the levels of NT-proBNP previously recorded in the general, healthy population, NT-proBNP concentrations were significantly elevated in the study cohort. Dialysis reduced NT-proBNP concentration by a mean of $39 \%$.

During the mean follow-up period of about 2 years, 34 patients died ( 9 deaths resulted from cardiac events). Regression analysis revealed significant inverse correlations between NTproBNP levels and left ventricular ejection fraction, urine production and $K t / V$, and a significant positive correlation between NT-proBNP levels and left ventricular hypertrophy. Patients with predialysis or postdialysis NT-proBNP levels above median cutoffs of $4,079 \mathrm{pg} / \mathrm{ml}$ and $2,759 \mathrm{pg} / \mathrm{ml}$, respectively, had a lower survival rate than did those with NT-proBNP concentrations below these thresholds. Notably, multivariate Cox regression analysis revealed only age and levels of NT-proBNP to be predictive of all-cause mortality.

Original article Madsen LH et al. (2007) N-terminal pro brain natriuretic peptide predicts mortality in patients with end-stage renal disease in hemodialysis. Kidney Int 71: 548-554

\section{Delaying dialysis until after uremic symptoms develop increases mortality risk}

The best time for people with end-stage renal disease to begin dialysis is unclear. Recommended glomerular filtration rates (GFRs) for starting dialysis range from $10 \mathrm{ml} / \mathrm{min}$ to $12.5 \mathrm{ml} / \mathrm{min}$, but patients sometimes refuse dialysis until after they develop major symptoms. A recent study investigated whether such delays affect clinical course and survival.

The investigators prospectively followed 233 patients in Hong Kong who were referred for peritoneal dialysis because their GFR was predicted to drop to $10 \mathrm{ml} / \mathrm{min}$ or less within 